\title{
Kinetic and Stationary Point-Set Embeddability for Plane Graphs ${ }^{\star}$
}

\author{
Zahed Rahmati, Sue H. Whitesides, and Valerie King \\ Department of Computer Science, University of Victoria, Canada \\ $\{$ rahmati, sue, val\}@uvic.ca
}

\begin{abstract}
We investigate a kinetic version of point-set embeddability. Given a plane graph $G(V, E)$ where $|V|=n$, and a set $P$ of $n$ moving points where the trajectory of each point is an algebraic function of constant maximum degree $s$, we maintain a point-set embedding of $G$ on $P$ with at most three bends per edge during the motion. This requires reassigning the mapping of vertices to points from time to time. Our kinetic algorithm uses linear size, $O(n \log n)$ preprocessing time, and processes $O\left(n^{2} \beta_{2 s+2}(n) \log n\right)$ events, each in $O\left(\log ^{2} n\right)$ time. Here, $\beta_{s}(n)=\lambda_{s}(n) / n$ is an extremely slow-growing function and $\lambda_{s}(n)$ is the maximum length of Davenport-Schinzel sequences of order $s$ on $n$ symbols.
\end{abstract}

Keywords: kinetic graph drawing, point-set embeddability, kinetic algorithm, plane graph.

\section{Introduction}

Problem Statement. Given a plane graph $G(V, E)$ on $n$ vertices and a point-set $P=$ $\left\{p_{1}, p_{2}, \ldots, p_{n}\right\}$, the problem of point-set embeddability without mapping is to draw $G$ on $P$ such that each vertex is mapped to a point of $P$ and such that no two edges intersect except at common vertices. A straight-line drawing is a drawing in which each edge of $G$ is mapped to a curve that is a line segment. A $k$-bend drawing is a drawing of $G$ such that each edge is mapped to a chain (curve) of at most $k+1$ line segments. The kinetic point-set embedding problem without mapping, with at most $k$ bends per edge, is to construct a $k$-bend drawing without mapping of $G$ on a set $P$ of $n$ moving points, where the trajectory of each point is an algebraic function of constant maximum degree $s$. In the kinetic setting, the trajectory of each point $p_{i}(t)=\left(x_{i}(t), y_{i}(t)\right)$ is defined by two polynomial functions of constant maximum degree $s$, and the objective is to maintain the embedding during the motion. During the time period, the point-set embedding may change or develop edge crossings, so a kinetic algorithm is needed to repair the embedding by remapping the vertices.

Related Work. Cabello [9] proved that deciding whether a planar graph $G$ can be embedded by straight-line edges without mapping onto a given set $P$ of points is NPcomplete, even when $G$ is 2-connected. Recently, Durocher and Mondal [13] showed that this problem is NP-complete for 3-connected planar graphs. Biedl and Vatshelle [6]

\footnotetext{
* Partially supported by NSERC and a University of Victoria Graduate Fellowship.
} 
proved that the problem is NP-hard for 2-connected outer-planar graphs if the embedding is fixed, 3-connected planar graphs of constant treewidth, and triangulated planar graphs. Gritzmann et al. [15] showed that the class of planar graphs such that all vertices are on the outer face (outerplanar graphs) is the largest class of graphs that can be embedded with straight-line edges without mapping onto any point set in general position (no three or more points collinear). There are algorithms for special cases in which the graph $G$ is a tree [8, 18] or an outerplanar graph [7, 15].

For $k$-bend drawing without mapping, Kaufmann and Wiese [19] gave a 1-bend drawing algorithm for 4-connected plane graphs and a 2-bend drawing algorithm for general plane graphs; for general graphs, their algorithm takes time $O(n \log n)$ (resp. $O\left(n^{2}\right)$ ) to draw a point-set embedding with at most three (resp. two) bends per edge. In particular, their algorithm draws a 3-bend drawing in $O(n \log n)$ time and then spends $O\left(n^{2}\right)$ time, using rotation, to transfer the 3-bend drawing to a 2-bend drawing. In addition, they proved that deciding whether there is a mapping such that each edge has at most one bend is NP-complete. Giacomo et al. [14] presented an $O(n \log n)$-time algorithm which improves the previous $O\left(n^{2}\right)$-time algorithm by Kaufmann and Wiese [19] and guarantees that no rotation is needed to obtain a 2-bend drawing.

Kinetic algorithms can model real-world phenomena where objects move with predictable trajectories in the short term, but may be subject to unpredictable changes in trajectory in the long term [2]. Basch, Guibas and Hershberger [5] introduced the kinetic data structure $(K D S)$ framework to handle such situations. Using this framework, one can maintain a target attribute of a graph on a set of moving points, where the trajectory of each point $p_{i}(t)=\left(x_{i}(t), y_{i}(t)\right)$ is defined by two algebraic functions of fixed degree. Kinetic versions of proximity graph problems have been studied extensively in the past decade. The vertices of proximity graphs represent points and the edges represent geometric relations between points. Kinetic studies of such graphs include Delaunay triangulations, Pie Delaunay graphs, and Euclidean minimum spanning trees [1, 16, 21].

To our knowledge there are no previous results for point-set embeddability of a plane graph on a set of points moving along predictable trajectories. While we currently have no particular application in mind, we believe that kinetic graph drawing is of inherent and compelling interest. We investigate the problem as follows. We find a Hamiltonian cycle of the graph $G$ and then map the Hamiltonian cycle to the set of points $P$. For each edge $v_{i} v_{j}$ of $G$, we draw a curve of line segments such that the slopes of the line segments are defined based on the difference between the subscripts $i$ and $j$ and the maximum slope of the edges of the Hamiltonian cycle. This assignment prevents intersections between edges during the motion except at times when two points change the ordering of their $x$-coordinates.

Kinetic Framework. Given a point-set embedding of a plane graph $G(V, E)$, in order to maintain the embedding as the points move, we define a set of certificates verifying the correctness of the embedding and a priority queue containing the failure times of these certificates. When the failure time of a certificate is equal to the current time, we invoke an update mechanism that replaces the invalid certificate(s) with a new, valid one(s).

The set of all algorithms and data structures used for maintaining the point-set embedding is called a kinetic data structure $(K D S)$. For example, suppose we want to 


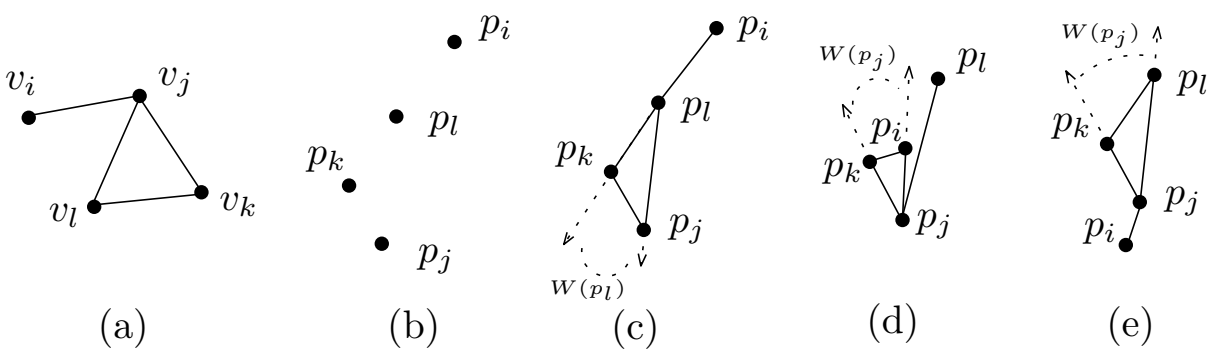

Fig. 1. (a) A plane graph $G(V, E)$ on vertices $v_{i}, v_{j}, v_{k}$, and $v_{l}$. (b) point set $P=\left\{p_{i}, p_{j}, p_{k}, p_{l}\right\}$. (c) A point-set embedding of $G$ on $P$ where $v_{i}, v_{j}, v_{k}$, and $v_{l}$ map to $p_{i}, p_{l}, p_{j}$, and $p_{k}$, respectively. (d) New configuration after $p_{i}$ moves inside the triangle $p_{j} p_{k} p_{l}$. (e) New configuration when $p_{l} p_{i}$ crosses the edge $p_{j} p_{k}$.

maintain the point-set embedding of the graph $G(V, E)$ in Figure 1 a on the set of points $P$ in Figure 1 $\mathrm{b}$, such that a point is outside the triangle created by the three other points; Figure 1 1 c depicts a drawing of $G$ before the points move. During the motion, assume no three or more points are collinear in any positive interval of time. Let $p_{j} p_{k} p_{l}$ be a triangle and let $W\left(p_{l}\right)$ be a wedge whose sides are created by removing the edge $p_{j} p_{k}$ and extending the two line segments $p_{l} p_{j}$ and $p_{l} p_{k}$. As shown in Figure 1 $1 \mathrm{c}$, the wedge $W\left(p_{l}\right)$ is the area between two half-lines $\overrightarrow{p_{l} p_{j}}$ and $\overrightarrow{p_{l} p k}$. To maintain a valid embedding of the plane graph $G$ as the points move in Figure 1 c, we create a certificate certifying that $p_{i}$ is outside the wedge $W\left(p_{l}\right)$; the correctness of this certificate, over a time interval, certifies the correctness of the point-set embedding of $G$ over that interval. Since we have the trajectory of the points, we can compute the (failure) time $t$ when the point $p_{i}$ moves inside the wedge $W\left(p_{l}\right)$. When the failure time of this certificate is equal to the current time $t$, then at time $t^{+}$, either point $p_{i}$ moves inside the triangle $p_{j} p_{k} p_{l}$ or the edge $p_{l} p_{i}$ intersects $p_{j} p_{k}$; in both cases, the point-set embedding is no longer valid. Therefore, at the critical time $t$, we replace the previous certificate with a new one and update the mapping of $G$ on $P$ at time $t^{+}$. When point $p_{i}$ crosses the half-line $\overrightarrow{p_{l} p_{j}}$ the new certificate should certify that the point $p_{l}$ is outside the wedge $W\left(p_{j}\right)$ created by half-lines $\overrightarrow{p_{j} p_{i}}$ and $\overrightarrow{p_{j} p_{k}}$; the updated point-set embedding is the triangle $p_{i} p_{j} p_{k}$ and the edge $p_{j} p_{l}$ outside the triangle in the case that $p_{i}$ moves inside the triangle $p_{j} p_{k} p_{l}$ (see Figure 1], d), or the triangle $p_{j} p_{k} p_{l}$ and the edge $p_{i} p_{j}$ outside the triangle otherwise (see Figure1 1e).

To measure the performance of a $K D S$ there are four generally accepted criteria, called efficiency, responsiveness, compactness, and locality, which we now describe [5]. When a certificate fails, that doesn't necessarily imply that the attribute, e.g., the validity of the point-set embedding, no longer holds. Events that don't change the target attribute are called internal events. Those events that produce changes in the target attribute are called external events; in Figure 1 c, the event is external. If the ratio between the number of the internal events and the number of the external events is polylogarithmic in the number of the points then the KDS is efficient. Typically, a $K D S$ processes some internal events. Indeed our kinetic 3-bend drawing algorithm which we present in Section 3 processes internal events as well as external events. 
If the processing time of an event is a polylogarithmic function of the number of points, the $K D S$ is responsive. The KDS is compact if its size is equal to the number of points, within a polylogarithmic factor. Each point $p_{i}$ has a trajectory, and the trajectory can change over time. When the trajectory changes, the certificates associated with the $p_{i}$ must change. If the number of all certificates associated with $p_{i}$ is a polylogarithmic function of the number of points, the $K D S$ is local. The locality criterion anticipates that, while points move with predictable trajectories in the short term, they may be subject to unpredictable changes in the long term.

Our Results. We provide a kinetic algorithm maintaining a 3-bend drawing without mapping of a given plane graph $G(V, E)$ on a set of $n=|V|$ moving points, where the trajectory of each point is an algebraic function of constant maximum degree $s$. Our KDS uses $O(n)$ size, $O(n \log n)$ preprocessing time, and processes $O\left(n^{2} \beta_{2 s+2}(n) \log n\right)$ events, each in $O\left(\log ^{2} n\right)$ time. Here, $\beta_{s}(n)=\frac{\lambda_{s}(n)}{n}$ is an extremely slow-growing function of $n$ and $\lambda_{s}(n)$ is the maximum length of Davenport-Schinzel sequences of order $s$ on $n$ symbols [20, 22]. In terms of the four standard KDS performance criteria, our KDS is efficient, responsive, local, and compact. In particular, in order to obtain a compact $K D S$, we provide a new $O(n \log n)$-time algorithm for point-set embedding of a given plane graph $G$ on a stationary point set $P$. This algorithm first draws a 3-bend drawing in $O(n \log n)$ time and then, spends linear time to transfer the 3-bend drawing to a 2-bend drawing in a way that guarantees that no rotation is needed to obtain the 2-bend drawing. In Section2, we introduce the main idea of constructing the 3-bend drawing and the 2-bend drawing without mapping and then, in Section 3 , we maintain them kinetically.

\section{$2 k$-Bend Drawing}

In this section we first show an $O(n \log n)$ algorithm for point-set embedding of a given plane graph $G$ on $P$ with at most three bends per edge and then, given this drawing, we provide a 2-bend drawing in linear time.

A maximal planar subdivision with vertex set $V$ is a triangulation, because no edge can be added without losing planarity. For any given plane graph $G(V, E)$, we add a set of edges $E^{\prime}$ to the graph $G$ to make it maximally planar, and then we embed the graph $G\left(V, E \cup E^{\prime}\right)$ on the set of given points $P$ and, finally, we remove the extra edges mapped from $E^{\prime}$. The remaining drawing is the point-set embedding of the graph $G(V, E)$ on the set of points $P$. Therefore, throughout this paper we assume the given plane graph $G$ is a triangulation.

\subsection{3-Bend Drawing}

We use a similar approach to that of Kaufmann and Wiese [19] to construct an initial drawing of the plane graph $G$ on a set of stationary points $P$. Our algorithm draws a point-set embedding with at most three bends per edge that we later extend to the kinetic setting. The key insight from [19] is finding a Hamiltonian cycle that has at least one edge, called an external edge, on the outer face of $G$. A Hamiltonian cycle can be found in linear time [14, 19] by adding dummy vertices; below we explain the approach of [19]. 
Each 4-connected planar graph is Hamiltonian [23]. In fact, any maximal planar graph with at most two separating triangles is Hamiltonian [10, 17, 24]; a separating triangle is a triangle whose removal separates the graph into more components. We now review how to construct a 4-connected graph from any planar graph. Using the algorithm by Chiba and Nishizeki [12], the separating triangles can be found efficiently. Kaufmann and Wiese [19] destroy the separating triangles by adding dummy vertices and edges to create a 4 connected graph. Then, using the algorithm of Chiba and Nishizeki [11], a Hamiltonian cycle of the 4connected graph can be found in linear time. Figure 2

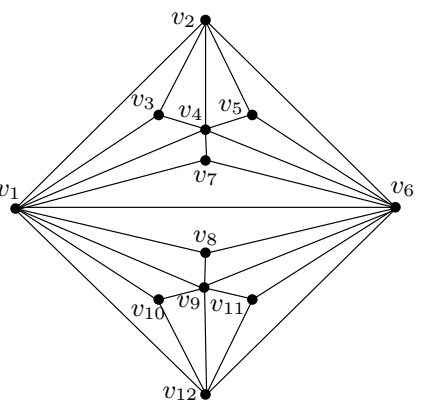

Fig. 2. A plane graph depicts a graph with separating triangles. Adding two new vertices $z_{1}$ and $z_{2}$ creates a new graph (see Figure 3 a) having a Hamiltonian cycle (bold edges); $z_{1}$ is placed on the edge $v_{1} v_{6}$ and partitions it into two edges $v_{1} z_{1}$ and $z_{1} v_{6}$ and creates two new edges $v_{7} z_{1}$ and $z_{1} v_{8}$; the corresponding dummy point of the dummy vertex $z_{1}$, in Figure $3, \mathrm{~b}$, is inserted in the middle of the segment $p_{7} p_{8}$. The new graph still has two separating triangles $v_{1} v_{2} v_{4}$ and $v_{2} v_{4} v_{6}$. For each dummy vertex $z_{k}$ we add a dummy point to the given set of points $P=\left\{p_{1}, p_{2}, \ldots, p_{n}\right\}$. The dummy vertex is placed on an edge $v_{i} v_{j}$ of the given graph $G$ and partitions $v_{i} v_{j}$ into two edges $v_{i} z_{k}$ and $z_{k} v_{j}$. The corresponding dummy point is inserted in the middle of the segment $p_{i} p_{j}$. Let $m$ be the number of dummy vertices, let $C=\left(u_{1}, u_{2}, \ldots, u_{n+m}, u_{1}\right)$ be the ordered vertices of the Hamiltonian cycle with external edge $e=u_{1} u_{n+m}$, and let chain $Q=\left\{q_{1}, q_{2}, \ldots, q_{n+m}\right\}$ be the list of $P$ plus the dummy points sorted in increasing order by their $x$-coordinates. In particular, for two points $q_{i}=\left(x_{i}, y_{i}\right)$ and $q_{j}=\left(x_{j}, y_{j}\right)$, if $i<j$ then $x_{i}<x_{j}$; note that no two points have the same $x$-coordinate. Call the edges on the Hamiltonian cycle of the plane graph $G$ hull edges, the edges inside the Hamiltonian cycle interior edges, and the edges outside of the Hamiltonian cycle exterior edges.

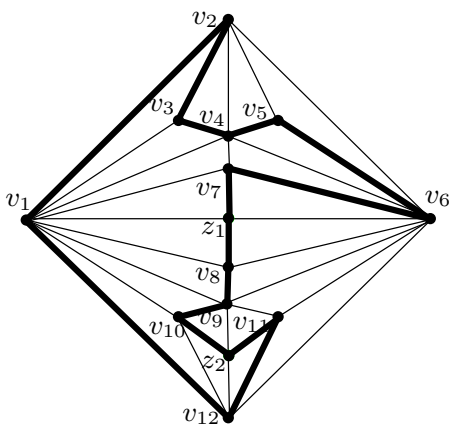

(a)

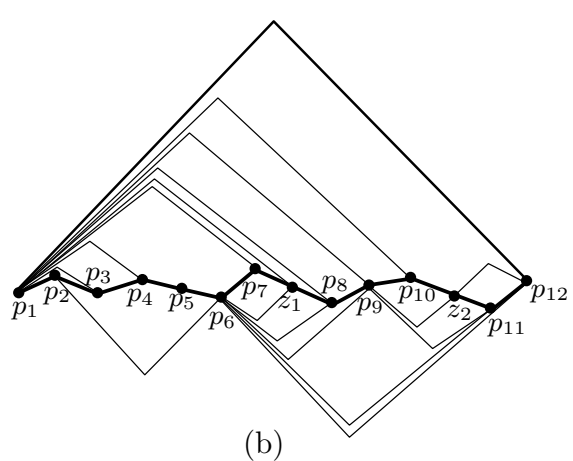

(b)

Fig. 3. (a) Adding two dummy vertices $z_{1}$ and $z_{2}$ and new edges creates a plane graph with a Hamiltonian cycle (bold edges). (b) A 3-bend drawing of $G$. 
In order to support kinetic drawing, we assign different slopes to the edges than does the algorithm of Kaufmann and Wiese [19]; our slopes prevent intersections between interior edges during the motion of the points; see Section 3 . Let $\delta$ be the maximum absolute slope of the edges of the chain $Q$. In particular, $\delta=\max _{i}\left|\frac{y_{i+1}-y_{i}}{x_{i+1}-x_{i}}\right|$ where $q_{i}=\left(x_{i}, y_{i}\right)$ and $q_{i+1}=\left(x_{i+1}, y_{i+1}\right)$ are consecutive edges of the chain $Q$. To draw the point-set embedding we map the hull edge $u_{i} u_{i+1}$ to the edge $q_{i} q_{i+1}$. For the external Hamiltonian edge $u_{1} u_{n+m}$ and each interior edge $u_{i} u_{j}$, where $i<j$, we also draw an edge with one bend $b_{i j}$ at the intersection of two lines, one through $u_{i}$ with slope $\left(1+\frac{j-i}{n+m}\right) \delta$ and the other one through $u_{j}$ with slope $-\left(1+\frac{j-i}{n+m}\right) \delta$; the mapping of the edge $u_{i} u_{j}$ is $q_{i} b_{i j} q_{j}$ which has one bend at $b_{i j}$. The interior edges are drawn above the chain $q_{1}, q_{2}, \ldots, q_{n+m}$ and the exterior edges are drawn in a similar way below the chain.

Theorem 1. The above point-set embedding of plane graph $G(V, E)$ onto the set of $n=|V|$ points $P$ is crossing-free, has at most three bends per edge, and is constructed in $O(n \log n)$ time.

Proof. After sorting the set of points $P$ by $x$-coordinates in $O(n \log n)$ time, we map the hull edges to edges of the chain $Q$ plus $q_{1} q_{n+m}$. This chain separates the interior edges and the exterior edges and it prevents intersections between these two types. In the following, we consider whether there are intersections among the interior edges; the proof that there is no intersection among the exterior edges is analogous. Let $q_{i} q_{j}$ and $q_{k} q_{l}$ be two interior edges; w.l.o.g., assume $i \leq k$. There are two possible situations: either $j \leq k$, in which case it is obvious that edge $q_{i} b_{i j} q_{j}$ doesn't cross edge $q_{k} b_{k l} q_{l}$ (Figure 3 b, see $p_{2} p_{6}$ and $p_{6} p_{8}$ ), or $j>k$, which implies that $j \geq l$ because the embedded plane graph $G$ has no edge crossing (edge $u_{i} u_{j}$ doesn't cross edge $u_{k} u_{l}$ ). In the second case $(i \leq k<l \leq j)$, the slope of $q_{i} b_{i j}\left(\right.$ resp. $\left.b_{i j} q_{j}\right)$ is $\left(1+\frac{j-i}{n+m}\right) \delta\left(\operatorname{resp} .-\left(1+\frac{j-i}{n+m}\right) \delta\right)$ which is sharper than the slope of $q_{k} b_{k l}$ (resp. $b_{k l} q_{l}$ ) because $j-i>l-k$; therefore, $b_{i j}$ is above $b_{k l}$ which means that edge $q_{i} b_{i j} q_{j}$ doesn't cross edge $q_{k} b_{k l} q_{l}$ (Figure 3., see $p_{1} p_{3}$ and $\left.p_{1} p_{4}\right)$. By an argument similar to one in [19], it can be proved that each edge of the graph $G$ is mapped to a chain of at most four line segments.

\subsection{2-Bend Drawing}

Let $q_{k}$ be a dummy point and let $q_{i} b_{i k} q_{k} b_{k j} q_{j}$ be a drawing of the edge $u_{i} u_{j}$ of $G$ that has three bends at $b_{i j}, q_{k}$, and $b_{k j}$. To reduce the number of bends to at most two bends per edge we replace the chain $b_{i k} q_{k} b_{k j}$ with a vertical line segment through $q_{k}$ [19], saving a bend at $q_{k}$, see Figure 4] The new edge $q_{i} q_{k}$ may cross other edges and destroy the planarity, see Figure 4, b, and so we need new assignments for the slopes of the edges in the point-set embedding. Let $r_{i j}=[i, j]$ denote the range of subscripts for the edge $q_{i} q_{j}$. The idea

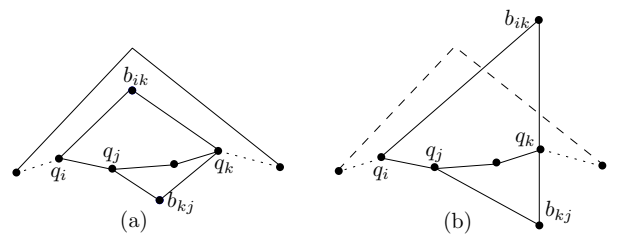

Fig. 4. Saving a bend at the dummy point $q_{k}$ behind our 2-bend drawing algorithm is 
to find the edges whose ranges contain the range $r_{i j}$, for all edges $q_{i} q_{j}$; if $r_{k l}$ covers $r_{i j}$ we assign slopes to segments of $q_{k} b_{k l} q_{l}$ so that they don't intersect the segments of $q_{i} b_{i j} q_{j}$. To store these nested layers of ranges we construct a nested tree $\mathcal{T}$ data structure. Each node $n_{i j}$ of $\mathcal{T}$ corresponds to an edge $q_{i} q_{j}$ and the subtree rooted at $n_{i j}$ stores all ranges covered by $r_{i j}$. Figure 5 b shows the nested tree $\mathcal{T}$ for the graph in Figure 5 a.

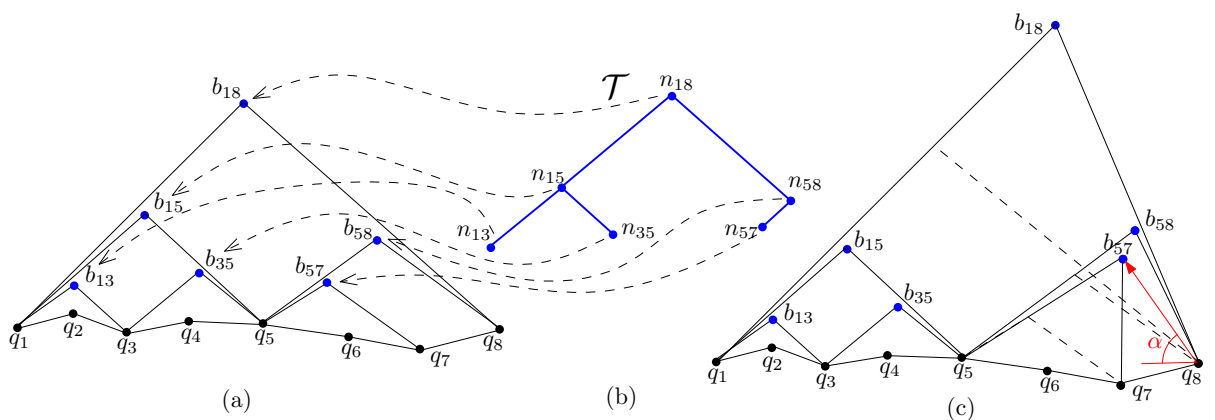

Fig. 5. (a) A 3-bend drawing. (b) The nested tree $\mathcal{T}$. (c) The 2-bend drawing.

\section{Lemma 1. Tree $\mathcal{T}$ can be built in $O(n)$ time from a 3-bend per edge drawing.}

Proof. Using a stack we construct the nested tree $\mathcal{T}$. For the external Hamiltonian edge $q_{1} q_{n+m}$, we push $q_{1}$ onto the stack, create a node $n_{1 n+m}$ as the root of $\mathcal{T}$ and a pointer pointing to this node. We process the endpoints of edges in order of increasing $x$-coordinate; if there are two or more edges incident to the same point, then we process these edges by decreasing order of their corresponding ranges. If we encounter the first endpoint of an edge $q_{i} q_{j}$, where $i<j$, we push the point $q_{i}$ and insert into $\mathcal{T}$ a new rightmost child of the node to which the pointer points; after this the pointer must point to the newly created node. If we encounter the second endpoint $q_{j}$ of $q_{i} q_{j}$, clearly, the top of the stack is the first endpoint $q_{i}$ and we pop the point $q_{i}$ and make the pointer point to the parent of the node $n_{i j}$ (in Figure 5 a, after creating the root $n_{18}$, first we see the point $q_{1}$ of the edge $q_{1} q_{5}$, whose range $r_{15}$ includes the range $r_{13}$ and so we create the node $n_{15}$ as the rightmost child of $n_{18}$; the pointer then points to $n_{15}$. After encountering the point $q_{1}$ of the edge $q_{1} q_{3}$ and creating the node $n_{13}$, the pointer points to the node $n_{13}$. Here, there are three $q_{1}$ 's in the stack corresponding to the three edges $q_{1} q_{8}, q_{1} q_{5}$, and $q_{1} q_{3}$. When we encounter $q_{3}$ we pop its corresponding $q_{1}$ and make the pointer point to $n_{15}$; continuing this process gives the nested tree $\mathcal{T}$ in Figure $5 \mathrm{~b}$ ). The running time is clear.

By traversing the nested tree $\mathcal{T}$ from the leaves to the root we show how to draw a 2-bend drawing. For each node $n_{i j}$ of $\mathcal{T}$ corresponding to the edge $q_{i} q_{j}$ we store two values $\delta_{l}$ and $\delta_{r}$; the slopes of $q_{i} b_{i j}$ and $b_{i j} q_{j}$ will be generated from $\delta_{l}$ and $\delta_{r}$. Let $n_{k l}$ be the parent of the node $n_{i j}$ corresponding to the edge $q_{k} q_{l}$. If $n_{i j}$ is a leaf and neither endpoint of $q_{i} q_{j}$ is a dummy point we set $\delta_{l}=\delta_{r}=\delta$ and set the slopes of $q_{i} b_{i j}$ and $b_{i j} q_{j}$ equal to $\left(1+\frac{j-i}{n+m}\right) \delta_{l}$ and $-\left(1+\frac{j-i}{n+m}\right) \delta_{r}$, respectively. If $q_{j}$ (resp. $q_{i}$ ) is a dummy point we set $\delta_{l}=\delta$ (resp. $\delta_{r}=\delta$ ); then, $b_{i j}$ is the intersection of the 
vertical line through $q_{j}$ (resp. $q_{i}$ ) and the line through $q_{i}$ with slope $\left(1+\frac{j-i}{n+m}\right) \delta_{l}$ (resp. $\left.-\left(1+\frac{j-i}{n+m}\right) \delta_{r}\right)$. To assign slopes to the edges whose ranges cover the range $r_{i j}$, we find the slope $\alpha$ of the line through $q_{l}$ (resp. $q_{k}$ ) and $b_{i j}$ (see Figure 5. c) and set $\delta_{r}=|\alpha|$ (resp. $\delta_{l}=\alpha$ ). After assigning the slopes $\delta_{l}$ and $\delta_{r}$ to all leaves we can find the slopes of the edges corresponding to the internal nodes of $\mathcal{T}$. For each internal node $n_{i j}$ we set $\delta_{l}$ (resp. $\delta_{r}$ ) to be the maximum of the $\delta_{l}$ 's (resp. $\delta_{r}$ 's) of the children of the node $n_{i j}$; the slope of $q_{i} b_{i j}$ (resp. $b_{i j} q_{j}$ ) is $\left(1+\frac{j-i}{n+m}\right) \delta_{l}$ (resp. $-\left(1+\frac{j-i}{n+m}\right) \delta_{r}$ ). If one of the endpoints of the edge $q_{i} q_{j}$ corresponding to the internal node $n_{i j}$ is a dummy point we handle $n_{i j}$ as we did for a leaf.

The above process assigns slopes to the edges such that if the range $r_{k l}$ covers the range $r_{i j}$ then the slope of $q_{k} b_{k l}$ (resp. $b_{k l} q_{l}$ ) is sharper than the slope of $q_{i} b_{i j}$ (resp. $b_{i j} q_{j}$ ) except when one of the endpoints of $q_{i} q_{j}$, say $p_{j}$, is a dummy point; in this case as explained above, we compute the slope $\alpha$ and use it to assign a valid slope to $b_{k l} q_{l}$ so that it does not intersect $b_{i j} q_{j}$. Therefore, $q_{i} q_{j}$ doesn't cross $q_{k} q_{l}$. Lemma1 together with the fact that traversing the nodes of the tree $\mathcal{T}$ takes linear time yields the following theorem.

Theorem 2. Given a 3-bend drawing of the plane graph $G$ on $P$, which can be constructed in $O(n \log n)$ time, a 2-bend drawing of $G$ on $P$ can be constructed in linear time.

\section{The Kinetic Drawing}

Now, we kinetically maintain the drawing of Section 2. We give a $K D S$ for maintaining the edges above the chain $q_{1}, q_{2}, \ldots, q_{n+m}$; the lower part can be maintained analogously.

\subsection{Kinetic 3-Bend Drawing}

In the 3-bend drawing of Section 2.1, each edge $q_{i} q_{j}$ above the chain is defined by two line segments $q_{i} b_{i j}$ and $b_{i j} q_{j}$ with positive slope $\left(1+\frac{j-i}{m+n}\right) \delta$ and negative slope - $(1+$ $\left.\frac{j-i}{m+n}\right) \delta$, respectively, where $\delta=\max _{i}\left|\frac{y_{i+1}-y_{i}}{x_{i+1}-x_{i}}\right|$. To maintain the point-set embedding over time, we maintain the maximum slope $\delta$ with a tool called the kinetic tournament tree [4]. We now describe it. Let $\widetilde{Q}$ be a set of moving objects such that each object has a time-varying value. The goal is to maintain over time the object with the maximum value. The kinetic tournament tree is a balanced binary tree that stores the objects at its leaves in an arbitrary order; each internal node stores the object with the maximum value of its two children. The root of the tree maintains the object in $\widetilde{Q}$ with the maximum value. For example, suppose we want to maintain the lowest point among a set of $n$ moving points along the $y$-axis such that each point moves according to an algebraic function. We can use a kinetic tournament tree with the points at the leaves; the root of the tree maintains the lowest point during the time intervals between critical events where the ordering changes. 
Here, we store the edges of the chain $q_{1}(t), q_{2}(t), \ldots, q_{n+m}(t)$ at the leaves of a kinetic tournament tree $\mathcal{K} \mathcal{T}$. At each internal node of $\mathcal{K} \mathcal{T}$, we store the winner edge of the two children, i.e., the edge having the larger absolute slope. We also define a certificate certifying the winner is steeper than the other edge; the failure time of the certificate is the time when the other edge becomes the winner. This event is called a tournament event. When a tournament event at an internal node occurs, we apply the changes from the internal node to the root of the tournament tree, in $O(\log n)$ time, so that the edge at the root of the tree always has the steepest slope among all edges of the chain $Q$.

We maintain a list $L_{\mathcal{Q}}$ of the set of points $Q$ sorted by increasing order of their $x$ coordinates. When the points move the order of the $x$-coordinates of two consecutive points may change. Let $q_{i^{\prime}}, q_{i}, q_{j}$, and $q_{j^{\prime}}$ be four consecutive points of $L_{\mathcal{Q}}$. For $q_{i}$ and $q_{j}$, we maintain a certificate certifying that the $x$-coordinate of $q_{i}$ is smaller than the $x$-coordinate of $q_{j}$; the failure time of the certificate is the time $t$ when $x_{i}(t)=x_{j}(t)$ and the order is changed at time $t^{+}$. Call this an order event. Whenever an order event between $q_{i}$ and $q_{j}$ occurs, we delete two edges $q_{i^{\prime}} q_{i}$ and $q_{j} q_{j^{\prime}}$ from $\mathcal{K} \mathcal{T}$ and add two new edges $q_{i^{\prime}} q_{j}$ and $q_{i} q_{j^{\prime}}$ into $\mathcal{K} \mathcal{T}$ (see Figure 6). Thus, we need a dynamic version of the kinetic tournament tree $\mathcal{K} \mathcal{T}$, called a dynamic kinetic tournament tree $(\mathcal{D K} \mathcal{T})[3]$, which supports insertions and deletions. The following theorem gives the construction time for a $\mathcal{D} \mathcal{K} \mathcal{T}$ and bounds the total number of events in this tournament.

Theorem 3. [3] A dynamic kinetic tournament tree $\mathcal{D} \mathcal{K} \mathcal{T}$, with a sequence of $m$ insertions and deletions whose maximum size at any time is $n$ (assuming $m \geq n$ ), generates at most $O\left(m \beta_{s+2}(n) \log n\right)$ events. Processing a tournament event takes $O\left(\log ^{2} n\right)$ time, and the $\mathcal{D} \mathcal{K} \mathcal{T}$ can be constructed in $O(n)$ time.

Since the trajectory of each point is an algebraic function of constant degree at most $s$, the Euclidean length of each edge of the chain is an algebraic function of constant degree at most $2 s$. Thus, the number of swaps between consecutive points in the sorted list $L_{\mathcal{Q}}$ is quadratic, and Theorem 3 implies the following.

Lemma 2. $\mathcal{D} \mathcal{K} \mathcal{T}$ can be constructed in linear time; the number of tournament events is $O\left(n^{2} \beta_{2 s+2}(n) \log n\right)$, and each event can be handled in $O\left(\log ^{2} n\right)$ time.

In addition to the sorted list $L_{\mathcal{Q}}$ and $\mathcal{D} \mathcal{K} \mathcal{T}$, we need a priority queue, called an event quеие, to maintain the failure times of the tournament events and the order events. Since the number of dummy vertices is $m=O(n)$, the sizes of the event queue and $\mathcal{D} \mathcal{K} \mathcal{T}$ are linear.

Lemma 3. The proposed $K D S$ (consisting of $L_{\mathcal{Q}}, \mathcal{D K} \mathcal{T}$, and the event queue) uses linear space.

Let $\operatorname{Inc}\left(q_{i}\right)$ be the set of internal edges incident to $q_{i}$. Whenever the time of the next event in the queue is equal to the current time, we update the KDS and replace the failure certificates with new ones. When an order event between $q_{i}$ and $q_{j}$ occurs, edges in the set $\operatorname{Inc}\left(q_{i}\right)$ (if non-empty) cross edges in the set $\operatorname{Inc}\left(q_{j}\right)$ (if non-empty), see Figure 6, b. To remove the edge crossings and restore the embedding we allocate $\operatorname{Inc}\left(q_{i}\right)$ to the point $q_{j}$ and vice versa, see Figure 6 c. Then, we delete the order certificates corresponding to $q_{i}$ and $q_{j}$ from the event queue and replace them with new ones certifying 


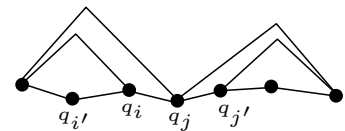

(a)

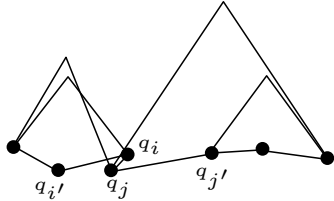

(b)

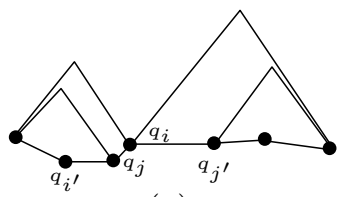

(c)

Fig. 6. (a) Before changing the ordering of $q_{i}$ and $q_{j}$. (b) After points $q_{i}$ and $q_{j}$ change their order. (c) Allocating $\operatorname{Inc}\left(q_{i}\right)$ to the point $q_{j}$ and vice versa.

that the order of the $x$-coordinates of $q_{i^{\prime}}, q_{j}, q_{i}$, and $q_{j^{\prime}}$ is in increasing order; the failure times are the times when two consecutive points change their $x$-coordinate ordering.

Theorem 4. Given an initial point-set embedding of a plane graph $G=(V, E)$ with at most three bends per edge on a set $P$ of $n=|V|$ points, where the trajectory of each point is a known algebraic function of constant degree at most $s$, there is a kinetic data structure KDS that maintains the embedding and that satisfies the following properties. The KDS has linear size, and processes $O\left(n^{2} \beta_{2 s+2}(n) \log n\right)$ tournament events and $O\left(n^{2}\right)$ order events, each in $O\left(\log ^{2} n\right)$ time. The KDS is efficient, responsive, compact and local.

Proof. The time required to apply a constant number of changes in the queue is $O(\log n)$. When an order event occurs a constant number of insertions and deletions is made in $\mathcal{D K} \mathcal{T}$; each one takes $O\left(\log ^{2} n\right)$ time (see Lemma 2). The compactness and the responsiveness, which depend on the number of events and the time to process them, respectively, follow from Lemmas 2 and 3

When a tournament event occurs, the winner stored at an internal node changes and the event may change the maximum slope $\delta$. The only type of event that may cause edges of the drawing to cross is the order event. The ratio between the number of internal events (tournament events) and the number of external events (order events) is poly$\operatorname{logarithmic}$ in $n$, so the $K D S$ is efficient. Each order event involves a constant number of other order events and $O(\log n)$ tournament events, so the number of all certificates associated with a particular point is polylogarithmic in $n$. Hence, the proposed KDS is local.

\subsection{Kinetic 2-Bended Drawing}

Recall from Section 2.2 that the slopes of edge $q_{i} q_{j}$, for a 2-bend per edge drawing, arise from two values $\delta_{l}$ and $\delta_{r}$ stored at node $n_{i j}$. In order to maintain these values in the kinetic setting we define two dynamic kinetic tournament trees $\mathcal{D} \mathcal{K} \mathcal{T}_{l}$ and $\mathcal{D} \mathcal{K} \mathcal{T}_{r}$ whose nodes store $\delta_{l}$ 's and $\delta_{r}$ 's, respectively, of the children of node $n_{i j}$; the root of $\mathcal{D K} \mathcal{T}_{l}$ (resp. $\mathcal{D} \mathcal{K} \mathcal{T}_{r}$ ) stores the larger value $\delta_{l}$ (resp. $\delta_{r}$ ) of the children.

Let $n_{k l}$ be the parent of node $n_{i j}$ and let $\mathcal{P}_{k l}$ be the path from $n_{k l}$ to the root of the nested tree $\mathcal{T}$. When the root of the tournament tree $\mathcal{D} \mathcal{K} \mathcal{T}_{l}$ or $\mathcal{D} \mathcal{K} \mathcal{T}_{r}$ changes, one of the children of $n_{k l}$ is deleted and a new one is inserted; thus, an insertion and a deletion are done in the corresponding tournament tree of $n_{k l}$; this tournament tree may cause 
an insertion and a deletion in the parent of $n_{k l}$ and hence updates to all corresponding tournament trees on the path $\mathcal{P}_{k l}$. When two points $q_{i}$ and $q_{j}$ change their $x$-coordinate ordering, we update the values $\delta_{l}$ and $\delta_{r}$ of the nodes corresponding to these points, up to the root of $\mathcal{T}$. Using this process we obtain a compact $K D S$ for 2-bend drawing.

\section{Conclusion}

We have introduced the investigation of graph drawing on moving points. In particular, we have introduced the kinetic data structure framework for maintaining a point-set embedding of a plane graph $G$ on a set $P$ of moving points, where each point moves according to an algebraic function of constant degree. We described an efficient kinetic algorithm for maintaining an embedding of $G$ with at most three bends per edge. In terms of the standard evaluation criteria for a $K D S$ framework, our $K D S$ for maintaining a drawing for $G$ with at most three bends per edge is efficient, responsive, local, and compact. We also gave a new $O(n \log n)$-time algorithm for point-set embedding with at most two bends per edge; we can kinetically maintain the 2-bend drawing but while this $K D S$ is compact, it does not satisfy the other three performance criteria. Therefore, future directions for this research include finding a $K D S$ for point-set embedding with at most two bends per edge that satisfies all four performance criteria and finding a $K D S$ for straight-line drawings for some special graphs like outerplanar graphs and trees.

\section{References}

[1] Abam, M.A., Rahmati, Z., Zarei, A.: Kinetic Pie Delaunay Graph and Its Applications. In: Fomin, F.V., Kaski, P. (eds.) SWAT 2012. LNCS, vol. 7357, pp. 48-58. Springer, Heidelberg (2012)

[2] Agarwal, P.K., Eppstein, D., Guibas, L.J., Henzinger, M.R.: Parametric and kinetic minimum spanning trees. In: FOCS, pp. 596-605. IEEE Computer Society (1998)

[3] Agarwal, P.K., Kaplan, H., Sharir, M.: Kinetic and dynamic data structures for closest pair and all nearest neighbors. ACM Trans. Algorithms 5, 4:1-4:37 (2008)

[4] Basch, J.: Kinetic data structures. PhD Thesis, Stanford University (1999)

[5] Basch, J., Guibas, L.J., Hershberger, J.: Data structures for mobile data. In: Proceedings of the 8th Annual ACM-SIAM Symposium on Discrete Algorithms, SODA 1997, pp. 747756. Society for Industrial and Applied Mathematics (1997)

[6] Biedl, T., Vatshelle, M.: The point-set embeddability problem for plane graphs. In: Proceedings of the 28th Symposuim on Computational Geometry, SoCG 2012, pp. 41-50. ACM, New York (2012)

[7] Bose, P.: On embedding an outer-planar graph in a point set. Comput. Geom. 23(3), 303312 (2002)

[8] Bose, P., McAllister, M., Snoeyink, J.: Optimal algorithms to embed trees in a point set. J. Graph Algorithms Appl. 1 (1997)

[9] Cabello, S.: Planar embeddability of the vertices of a graph using a fixed point set is NPhard. J. Graph Algorithms Appl. 10(2), 353-363 (2006)

[10] Chen, C.: Any maximal planar graph with only one separating triangle is hamiltonian. J. Comb. Optim. 7(1), 79-86 (2003)

[11] Chiba, N., Nishizeki, T.: The hamiltonian cycle problem is linear-time solvable for 4connected planar graphs. J. Algorithms 10(2), 187-211 (1989) 
[12] Chiba, N., Nishizeki, T.: Arboricity and subgraph listing algorithms. SIAM J. Comput. 14(1), 210-223 (1985)

[13] Durocher, S., Mondal, D.: On the Hardness of Point-Set Embeddability. In: Rahman, M. S., Nakano, S.-I. (eds.) WALCOM 2012. LNCS, vol. 7157, pp. 148-159. Springer, Heidelberg (2012)

[14] Giacomo, E.D., Didimo, W., Liotta, G., Wismath, S.K.: Curve-constrained drawings of planar graphs. Computational Geometry 30(1), 1-23 (2005)

[15] Gritzmann, P., Mohar, B., Pach, J., Pollack, R.: Embedding a planar triangulation with vertices at specified points. American Mathematical Monthly 98, 165-166 (1991)

[16] Guibas, L.J., Mitchell, J.S.B.: Voronoi Diagrams of Moving Points in the Plane. In: Schmidt, G., Berghammer, R. (eds.) WG 1991. LNCS, vol. 570, pp. 113-125. Springer, Heidelberg (1992)

[17] Helden, G.: Each maximal planar graph with exactly two separating triangles is hamiltonian. Discrete Applied Mathematics 155(14), 1833-1836 (2007)

[18] Ikebe, Y., Perles, M.A., Tamura, A., Tokunaga, S.: The rooted tree embedding problem into points in the plane. Discrete \& Computational Geometry 11, 51-63 (1994)

[19] Kaufmann, M., Wiese, R.: Embedding vertices at points: Few bends suffice for planar graphs. J. Graph Algorithms Appl. 6(1), 115-129 (2002)

[20] Nivasch, G.: Improved bounds and new techniques for Davenport-Schinzel sequences and their generalizations. J. ACM 57, 17:1-17:44 (2010)

[21] Rahmati, Z., Zarei, A.: Kinetic Euclidean minimum spanning tree in the plane. Journal of Discrete Algorithms 16, 2-11 (2012)

[22] Sharir, M., Agarwal, P.K.: Davenport-Schinzel Sequences and their Geometric Applications. Cambridge University Press, New York (2010)

[23] Tutte, W.: A theorem on planar graphs. Trans. Amer. Math. Soc. 82, 99-116 (1956)

[24] Whitney, H.: A theorem on graphs. Ann. Math. 32, 378-390 (1931) 